\title{
Postexposure prophylaxis with inhaled zanamivir was efficacious for household contacts of people with influenza
}

\author{
Hayden FG, Gubareva LV,Monto ASet al., for the Zanamivir Family Study Group. Inhaled zanamivir for the prevention of \\ influenza in families. N Engl J Med 2000 Nov 2;343:1282-3.
}

\section{QUESTION: In household contacts of people with influenza, what is the efficacy of postexposure prophylaxis with inhaled zanamivir?}

\section{Design}

Randomised \{allocation concealed*\}†, blinded \{patients, clinicians, outcome assessors, and statisticians $\}+, *$ controlled trial with follow up to 28 days.

\section{Setting}

15 centres in the US, Canada, the UK, and Finland.

\section{Participants}

337 families with $2-5$ members, including $\geqslant 1$ adult and $\geqslant 1$ child who was 5-17 years of age. Family members who were hypersensitive to zanamivir, immunocompromised, pregnant, breast feeding, or using an anti-influenza agent were not assigned to a study drug. All families were included in the intention to treat analysis, and 1147 of $1158(99 \%)$ participants completed the study.

Source of funding: Glaxo Wellcome.

For correspondence: Dr F G Hayden, University of Virginia, Health Sciences Center Box 800473 ,

Charlottesville, VA 22908, USA.

\section{Intervention}

Within 36 hours after 1 family member contracted an influenza-like illness (index case), eligible family members began to take the study drug. Influenza-like illness was defined as $\geqslant 2$ of temperature $\geqslant 37.8^{\circ} \mathrm{C}$, feverishness, cough, headache, sore throat, and myalgia. The 321 index case patients (mean age 19 y, $58 \%$ women) were

Post-exposure zanamivir v placebo for prevention of influenza in household contacts of people with influenza-like illness:

\begin{tabular}{|c|c|c|c|}
\hline Outcome at 28 days & Zanamivir Placebo & RRR $(95 \%$ Cl) & NNT (Cl) \\
\hline $\begin{array}{l}\text { Families with laboratory confirmed } \\
\text { influenza in } \geqslant 1 \text { contact }\end{array}$ & $19 \%$ & $78 \%$ (53 to 90$)$ & 7 (5 to 12$)$ \\
\hline
\end{tabular}

$\ddagger$ Abbreviations defined in glossary; RRR, NNT, and $\mathrm{Cl}$ calculated from data in article.

allocated to inhaled zanamivir $(\mathrm{n}=163), 10 \mathrm{mg}$, or placebo $(n=158)$ twice a day for 5 days. The 837 other healthy family members (mean age 26 y, $55 \%$ women) received the same drug as the index case patient $(414$ received zanamivir, $10 \mathrm{mg}$ once daily for $10 \mathrm{~d}$, and 423 received placebo).

\section{Main outcome measure}

Proportion of families with $\geqslant 1$ initially healthy family member who developed symptomatic, laboratory confirmed influenza ( $\geqslant 3$ consecutive diary card entries listing $\geqslant 2$ of temperature $\geqslant 37.8^{\circ} \mathrm{C}$, feverishness, cough, headache, sore throat, and myalgia).

\section{Main results}

Intention to treat analysis showed that fewer families in the zanamivir group had $\geqslant 1$ household contact in whom symptomatic laboratory confirmed influenza developed than families in the placebo group (table). No resistant variants to zanamivir were detected. Median duration of symptoms in ill index case patients was shorter in the zanamivir group than in the placebo group $(5.0 v 7.5 \mathrm{~d}, \mathrm{p}=0.01)$.

\section{Conclusion}

Postexposure inhaled zanamivir prevented development of influenza in household contacts of family members with influenza-like illness who were also treated with inhaled zanamivir.

*See glossary.

$\dagger$ Information provided by author.

\section{COMMENTARY}

Although inactivated influenza vaccines remain the cornerstone of prevention for both influenza A and B, a new class of antiviral agents, the neuraminidase inhibitors, now have a role in the treatment and prevention of influenza.

Two neuraminidase inhibitors are licensed in the US. Zanamivir (administered by inhalation) is approved for treatment of influenza A and B. Oseltamivir phosphate (given orally) is approved for both treatment and chemoprophylaxis of influenza A and B. For both treatment of ill people and chemoprophylaxis of household contacts, the efficacy of the 2 drugs appears similar; although zanamivir has fewer side effects overall than oseltamivir, it does have the side effect of bronchospasm.

Neuraminidase inhibitors offer advantages over the current drugs, amantadine and rimantadine, in that they are effective against both influenza A and B and have fewer bothersome side effects. Their usefulness, however, is limited by cost; currently, the wholesale cost of neuraminidase inhibitors is about $\$ 50$ per 5 day course of treatment.

The side effects of neuraminidase inhibitors vary by route of administration. Treatment with zanamivir may exacerbate underlying airway disease. Other reported side effects include nausea, dizziness, diarrhoea, nasal symptoms, and other symptoms; none occurred in more than $5 \%$ of patients. About $10 \%$ of people treated with oseltamivir had nausea or vomiting. About $10 \%$ to $30 \%$ of people taking amantadine had central nervous system side effects (anxiety, nervousness, lightheadedness, and difficulty concentrating) compared with $<10 \%$ of those taking rimantadine.

These 2 studies indicate that neuraminidase inhibitors have a role in prevention, if only to provide a brief period for people to become immunised after the start of an influenza epidemic. Published recommendations for the preventive use of these drugs incorporate 2 main strategies: temporarily protecting high risk people who may not be immune after vaccination and institutional outbreak control. ${ }^{2}$ 


\title{
Oseltamivir reduced new cases of influenza in household contacts of people with clinical or confirmed influenza
}

\author{
Welliver R, Monto AS, Carewicz O, et al., for the Oseltamivir Post Exposure Prophylaxis Investigator Group. Effectiveness of \\ oseltamivir in preventing influenza in household contacts: a randomized controlled trial.JAMA 2001 Feb 14;285:748-54.
}

\section{QUESTION: In households with a member who has clinical or confirmed influenza,} does oseltamivir prevent the secondary spread of influenza to household contacts?

\section{Design}

Randomised \{allocation concealed $*\} \uparrow$, blinded \{patients, clinicians, outcome assessors, and statisticians $\} \nmid, *$ placebo controlled trial with follow up during the 7 day dosing period.

\section{Setting}

76 centres in North America and Europe.

\section{Participants}

374 households (962 potential contacts, 2 to 8 per household) that had 1 member with clinical or laboratory confirmed influenza (index case) identified within 48 hours of symptom onset (minimum cough and coryza). Households were excluded if they had members who were pregnant or nursing or who had cancer, immunosuppression, HIV infection, or chronic liver or renal disease or if they had elderly members with Mental Status Questionnaire scores $<7$. Children $<12$ years of age were excluded as contacts.

\section{Intervention}

During the influenza outbreak in the winter of 1998 to 1999, 195 households with 497 contacts were allocated to oseltamivir, $75 \mathrm{mg}$ once/day for 7 days, and 178 households with 465 contacts were allocated to placebo. The person in each household who was the index case did not receive antiviral treatment. Follow up of households and contacts was 99\%.

\section{Main outcome measure}

Household contacts with laboratory confirmed influenza.

\section{Main results}

Oseltamivir reduced the rate of new cases of laboratory confirmed influenza in household contacts of all index cases (ie, with or without confirmed influenza) $(n=377)$ and in contacts of index cases with laboratory confirmed cases of influenza $(\mathrm{n}=163)$ (table). Patients in the oseltamivir and placebo groups had similar rates of adverse gastrointestinal effects and withdrawals for adverse effects.

\section{Conclusion}

Oseltamivir reduced the incidence of influenza in household contacts of people with clinical and confirmed influenza.

*See glossary.

$\dagger$ Information provided by author.

Oseltamivir v placebo for prevention of laboratory confirmed influenza in household contacts of people with influenza $\$$

\begin{tabular}{|c|c|c|c|c|}
\hline $\begin{array}{l}\text { Outcomes during } 7 \text { day } \\
\text { dosing }\end{array}$ & Oseltamivir & Placebo & $\operatorname{RRR}(95 \% \mathrm{CI})$ & NNT (Cl) \\
\hline \multicolumn{5}{|l|}{ All index cases $(n=377)$} \\
\hline Individuals with influenza & $0.8 \%$ & $7.4 \%$ & $89 \%(71$ to 96$)$ & Not applicable \\
\hline Households with influenza & $2.1 \%$ & $14.6 \%$ & $86 \%(60$ to 95$)$ & $8(6$ to 14$)$ \\
\hline \multicolumn{5}{|c|}{ Index cases with confirmed influenza $(n=163)$} \\
\hline Individuals with influenza & $1.4 \%$ & $12.6 \%$ & $89 \%(67$ to 97$)$ & Not applicable \\
\hline Households with influenza & $3.6 \%$ & $22.8 \%$ & $84 \%$ (49 to 95$)$ & $6(4$ to 11$)$ \\
\hline
\end{tabular}

¥Abbreviations defined in glossary; NNTs and Cls calculated from data in article.
Source of funding: Hoffmann-La Roche.

For correspondence: Dr J S Oxford, St Bartholomew's and Royal London Hospital School of Medicine and Dentistry, The Medical Building, 327 Mile End Road,London E1 4NS, UK. Fax +44 (0)2078826990.

\section{COMMENTARY_continued from previous page}

The duration of treatment for either drug is 5 days, provided that the drug is started within 2 days of symptom onset. The duration of administration of the drug for chemoprophylaxis varies. If used as described in these studies for prevention of influenza in healthy people, the duration of administration is $7-10$ days. To prevent influenza among people with compromised immune systems who will not respond adequately to influenza vaccine, the drug should be given for the duration of influenza activity in the community.

The choice of antiviral drug depends on the strain of virus circulating, the known efficacy of the drug, expected side effects, and cost. To prevent influenza in people for whom immunisation may not be effective, an antiviral drug should be taken for an extended period. In these patients, the cost of neuraminidase inhibitors, which is higher than that of amantadine and rimantadine, may limit their acceptability.

Neuraminidase inhibitors can prevent the less serious complications of influenza, such as otitis and sinusitis. The information is insufficient to ascertain the degree to which these drugs reduce the most serious complications of influenza-pneumonia and death.

1 Couch RB. Prevention and treatment of influenza. N Engl J Med 2000;343:1778-87.

2 Bridges CB, Fukuda K, Cox JN, et al, for the Advisory Committee on Immunization Practices. Prevention and control of influenza. Recommendations of the advisory committee on immunization practices (ACIP). MMWR Morb Mortal Wkly Rep 2001;50(RR-4):1-44. 